\title{
Transducin Translocation in Rods Is Triggered by Saturation of the GTPase-Activating Complex
}

\author{
Ekaterina S. Lobanova, ${ }^{1}$ Stella Finkelstein, ${ }^{1}$ Hongman Song, ${ }^{2}$ Stephen H. Tsang, ${ }^{3}$ Ching-Kang Chen, ${ }^{4}$ Maxim Sokolov, ${ }^{5}$ \\ Nikolai P. Skiba, ${ }^{1}$ and Vadim Y. Arshavsky ${ }^{1}$ \\ ${ }^{1}$ Albert Eye Research Institute, Duke University Medical Center, Durham, North Carolina 27710, 2Department of Biochemistry, West Virginia University \\ School of Medicine, Morgantown, West Virginia 26506, ${ }^{3}$ Brown Glaucoma Laboratory, Edward Harkness Eye Institute, Columbia University, New York, \\ New York 10032, ${ }^{4}$ Department of Biochemistry, Virginia Commonwealth University, Richmond, Virginia 23298, and ${ }^{5}$ Sensory Neuroscience Research \\ Center and West Virginia University Eye Institute, Morgantown, West Virginia 26506
}

Light causes massive translocation of G-protein transducin from the light-sensitive outer segment compartment of the rod photoreceptor cell. Remarkably, significant translocation is observed only when the light intensity exceeds a critical threshold level. We addressed the nature of this threshold using a series of mutant mice and found that the threshold can be shifted to either a lower or higher light intensity, dependent on whether the ability of the GTPase-activating complex to inactivate GTP-bound transducin is decreased or increased. We also demonstrated that the threshold is not dependent on cellular signaling downstream from transducin. Finally, we showed that the extent of transducin $\alpha$ subunit translocation is affected by the hydrophobicity of its acyl modification. This implies that interactions with membranes impose a limitation on transducin translocation. Our data suggest that transducin translocation is triggered when the cell exhausts its capacity to activate transducin GTPase, and a portion of transducin remains active for a sufficient time to dissociate from membranes and to escape from the outer segment. Overall, the threshold marks the switch of the rod from the highly light-sensitive mode of operation required under limited lighting conditions to the less-sensitive energy-saving mode beneficial in bright light, when vision is dominated by cones.

Key words: transducin; photoreceptor; protein translocation; GTPase; vision; retina

\section{Introduction}

Light adaptation is the ability of photoreceptors to respond to a very large range of light intensity changes during a normal diurnal cycle. One of the mechanisms that contributes to light adaptation is the massive translocation of signaling proteins out of and into the outer segment, the photoreceptor compartment in which phototransduction takes place (for review, see Calvert et al., 2006). Transducin is one of the proteins that undergoes translocation after it is activated by photoexcited rhodopsin $\left(\mathrm{R}^{\star}\right)$ and becomes separated into individual $\alpha\left(\mathrm{G} \alpha_{\mathrm{t}}\right)$ and $\beta \gamma\left(\mathrm{G} \beta_{1} \gamma_{1}\right)$ subunits. A striking and unexplained feature of this phenomenon is that the majority of transducin translocates only when illumination reaches a critical threshold level (Sokolov et al., 2002).

One possibility is that this light intensity threshold reflects the

Received Nov. 17, 2006; revised Dec. 22, 2006; accepted Dec. 24, 2006.

This work was supported by National Institutes of Health (NIH) Grants EY10336 (V.Y.A.) and EY013811 (C.-K.C.) and NIH Core Grant for Vision Research EY5722 to Duke University. V.Y.A. is a recipient of a Senior Investigator Award from Research to Prevent Blindness. M.S. was supported by the NIH/National Center for Research Resources 2P20 RR15574-06 Center of Biomedical Research Excellence in Sensory Neuroscience. S.H.T. is a Fellow of the BurroughsWellcome Program in Biomedical Sciences and was also supported by the Foundation Fighting Blindness, American Geriatrics Society, Schneeweiss Foundation, Joel Hoffman Fund, Hirschl Charitable Trust, Eye Surgery Fund, and Bernard Becker Association of University Professors in Ophthalmology Research to Prevent Blindness Award. We thank S. A. Baker for critically reading this manuscript.

Correspondence should be addressed to Vadim Y. Arshavsky, Duke University Eye Center, 5008 Albert Eye Research Institute, 2351 Erwin Road, Durham, NC 27710. E-mail: vadim.arshavsky@duke.edu.

DOI:10.1523/JNEUROSCI.5010-06.2007

Copyright $\odot 2007$ Society for Neuroscience $\quad$ 0270-6474/07/271151-10\$15.00/0 intrinsic properties of the transducin activation/inactivation cycle, in which transducin activation is followed by its rapid inactivation by the GTPase-activating complex consisting of three proteins: regulator of G-protein signaling 9 (RGS9) G-protein $\beta$ subunit 5 (G $\beta 5$ ), and RGS9 anchor protein (R9AP) (for review, see Burns and Arshavsky, 2005). A recent study revealed that $\mathrm{G} \alpha_{\mathrm{t}}$ translocates at a lowered light intensity in mice in which its lifetime in the activated state is prolonged as a result of the knockout of RGS9 (Kerov et al., 2005). However, prolonged lifetime of activated $\mathrm{G} \alpha_{\mathrm{t}}$ also prolongs activation of the entire phototransduction cascade, increases the photoresponse duration, and saturates rods at dimmer light than normally (Chen et al., 2000). This suggests an alternative: that the threshold of transducin translocation originates from a critical activation level of signaling events downstream of transducin, such as dephosphorylation of phosducin (Lee et al., 2004), engagement of molecular motors (Peterson et al., 2005), or modulation of permeability of the connecting cilium through which transducin escapes from rod outer segments to other parts of the cell (Pulvermuller et al., 2002; Giessl et al., 2004).

To solve this dilemma, we compared light dependencies of transducin translocation in mice lacking RGS9 and W70A mice, in which the duration of transducin activity was also increased, but downstream signaling was severely impaired (Tsang et al., 1998). We found that the threshold of transducin translocation in both mice was shifted to dimmer light, suggesting that the thresh- 
old relates to the speed of transducin inactivation. We tested this directly in mice overexpressing RGS9 (Krispel et al., 2006) and found that the threshold was shifted to brighter light. These data suggest that the threshold for massive transducin translocation reflects the light intensity at which the amount of activated transducin exceeds the capacity of RGS9 to activate its GTPase. In the final series of experiments, we demonstrated that acylation of $\mathrm{G} \alpha_{\mathrm{t}}$ imposes a limitation on its mobility. The more hydrophobic the acyl group is, the lower the extent of transducin translocation from the outer segment is. Overall, the threshold could be viewed as a self-regulating mechanism switching the cell into a deeply light-adapted state in which the depletion of transducin in the outer segment causes the lower responsiveness of a rod to light and/or reduction in the metabolic stress under conditions of bright illumination, when mammalian vision is dominated by cones.

\section{Materials and Methods}

Animals. C57BL/6 pigmented wild-type mice and CD-1 albino wild-type mice were purchased from Charles River Laboratories (Wilmington, MA). R9AP knock-out mice were described by Keresztes et al. (2004), W70A mice were described by Tsang et al. (1998), and R9APoverexpressing mice were described by Krispel et al. (2006). All animals were housed in a $12 \mathrm{~h}$ day/night cycle and used between the ages of 2 and 3 months. Female Long-Evans rats (50-60 d of age) were purchased from Charles River Laboratories.

Antibodies. Antibodies precipitating $\mathrm{G} \alpha_{\mathrm{t}}$ were obtained at Elmira Biologicals (Iowa City, IA) by immunizing sheep with purified $\mathrm{G} \alpha \alpha_{\mathrm{t}}$. To obtain $\mathrm{G} \alpha_{\mathrm{t}}$, transducin heterotrimer was purified from frozen bovine retinas as described by Ting et al. (1993), and the $\mathrm{G} \alpha_{\mathrm{t}}$ subunit was separated from $\mathrm{G} \beta_{1} \gamma_{1}$ on a $1 \mathrm{ml}$ Blue Sepharose column (GE Healthcare, Piscataway, NJ) according to Heck and Hofmann (2001). Specific antibodies were purified from immune serum on an AminoLink Plus column (Pierce Biotechnology, Rockford, IL) containing covalently attached transducin heterotrimer, according to the instructions from Pierce Biotechnology. Rabbit phospho-specific antibodies against mouse phosducin phosphorylated at serine residues 54 and 71 were generated by 21st Century Biochemicals (Marlboro, MA), essentially as described by Lee et al. (2004), against synthetic phosphopeptides corresponding to amino acids $50-59$ and $67-77$ of mouse phosducin. The sheep antiphosducin antibody was described by Sokolov et al. (2004). Antirhodopsin monoclonal antibodies $4 \mathrm{D} 1$ were a generous gift from R. S. Molday (University of British Columbia, Vancouver, British Columbia, Canada). Anti-cytochrome $\mathrm{C}$ antibodies were obtained at Elmira Biologicals (Iowa City, IA) by immunizing sheep with whole bovine cytochrome C (Sigma, St. Louis, MO), followed by purification of specific antibodies on an AminoLink column containing covalently attached cytochrome C. Western blot analysis and immunolocalization of transducin subunits were performed using the SC-379 antibody against $\mathrm{G} \beta_{1}$ and the SC-389 antibody against $\mathrm{G} \alpha_{\mathrm{t}}$ [both from Santa Cruz Biotechnology (Santa Cruz, CA)]. The secondary antibodies used for immunolocalization were Alexa Fluor 594 conjugated with goat anti-rabbit antibodies (Invitrogen, San Diego, CA). Western blots were analyzed on the Odyssey Infrared Imaging System (LI-COR Biosciences, Lincoln, NE) using the secondary goat or donkey antibodies conjugated with Alexa Fluor 680 (Invitrogen).

Light adaptation of animals and determination of rhodopsin bleaching levels. Mice or rats were dark adapted for at least $12 \mathrm{~h}$ and anesthetized with an intraperitoneal injection of a ketamine/xylazine mixture (75/10 $\mathrm{mg} / \mathrm{kg}$, respectively). Their pupils were dilated with a mixture of $1 \%$ cyclopentolate- $\mathrm{HCl}$ and $2.5 \%$ phenylephrine. Light of desired duration ( $30 \mathrm{~min}$ for mice and $40 \mathrm{~min}$ for rats) and intensity was delivered to the eyes by a bifurcated fiber-optic guide from an adjustable light source equipped with a $100 \mathrm{~W}$ halogen bulb. Even illumination throughout the entire retina was achieved by positioning a small white dome between the light guide and each of the eyes, just above the cornea surface, with drops of Gonak (Akorn, Buffalo Grove, IL) sealing the eye surface from expo- sure to air and preventing cataract formation. The light intensity at the position corresponding to the eye surface was measured by a calibrated photodiode with a spectral sensitivity closely matched to that of rhodopsin (for a detailed description, see Sokolov et al., 2002). The rate of rhodopsin photoactivation in rods was determined from the amount of bleached rhodopsin after illumination by light of known intensity. This was achieved by measuring rhodopsin concentration in the retina homogenate by difference spectroscopy before and after rhodopsin regeneration with 11-cis-retinal according to the protocol described by Strissel et al. (2006). The rate of rhodopsin photoexcitation was calculated by dividing the fraction of bleached rhodopsin by duration of illumination and multiplying by the total amount of rhodopsin molecules in a mouse $\operatorname{rod}\left(7 \times 10^{7}\right)$ (Lyubarsky et al., 2004).

Immunolocalization of transducin subunits. Eyes were enucleated from dark- or light-adapted mice and fixed for $2 \mathrm{~h}$ with $4 \%$ formaldehyde in PBS buffer, pH 7.5 (Fisher Scientific, Hampton, NH). The anterior portion of the fixed eye was removed, and the eyecups were submersed in $30 \%$ sucrose in PBS for $2 \mathrm{~h}$ at $4^{\circ} \mathrm{C}$. The eyecups were kept for $1 \mathrm{~h}$ at $4^{\circ} \mathrm{C}$ in a 1:1 mixture of $30 \%$ sucrose in PBS and optimum cutting temperature (OCT) embedding medium (Sakura Finetek Japan, Tokyo, Japan), embedded in OCT, and frozen on dry ice. Cross sections (12 $\mu \mathrm{m})$ were collected on Superfrost Plus slides (Fisher Scientific), air dried overnight at room temperature, rinsed in PBS, permeabilized for 15 min with PBS containing $0.25 \%$ Triton X-100, washed with PBS, and incubated for $1 \mathrm{~h}$ with blocking solution containing 3\% normal goat serum in PBS in a humidified chamber. For detection of transducin, sections were incubated for $2 \mathrm{~h}$ with rabbit anti-G $\alpha_{\mathrm{t}}$ or anti-G $\beta_{1}$ antibody (1:1000 dilution each), washed three times with PBS, incubated for $1 \mathrm{~h}$ with goat antirabbit Alexa Fluor 594 secondary antibody (1:500), rinsed three times in PBS, mounted with Fluoromount G (Electron Microscopy Sciences, Hatfield, PA) under glass coverslips, dried and visualized using a Nikon (Tokyo, Japan) Eclipse 90i confocal microscope. For standardization of immunostaining, sections from the eyes of mutant and wild-type mice illuminated by identical light intensities were processed together on the same slides and visualized using the same microscope settings.

Phosducin phosphorylation assays. After light or dark adaptation, anesthetized mice were killed by cervical dislocation, and their eyes were rapidly frozen on dry ice. Each eye was homogenized in $0.2 \mathrm{ml}$ of buffer, containing 125 mm Tris/HCl, pH 6.8, 4\% SDS, 6 м urea, 10 mg/ml DTT, and $5 \mu \mathrm{M}$ phosphatase inhibitor microcystin-LR (Sigma), by short ultrasonic pulses delivered from a Microson ultrasonic cell disruptor equipped with a $3 \mathrm{~mm}$ probe (Misonix, Farmingdale, NY). This resulted in complete disintegration of the retina. Homogenates were cleared by centrifugation, and $20 \mu \mathrm{l}$ aliquots were separated on 18-well 10\% Tris$\mathrm{HCl}$ Criterion gels (Bio-Rad, Hercules, CA) and transferred to Immobilon-FL polyvinylidene difluoride (PVDF) membrane (Millipore, Billerica, MA). Phosphorylation of serine 54 and serine 71 was detected using phospho-site-specific antibodies and the Odyssey Infrared Imaging System (LI-COR Biosciences). To determine total phosducin content, homogenates were diluted 100 -fold, and $20 \mu \mathrm{l}$ aliquots were analyzed using sheep polyclonal antibody against phosducin. Fluorescence of phosphorylated phosducin bands was divided by fluorescence of total phosducin bands, and then the amounts of phosphorylated phosducin in the light-adapted samples were expressed as percentages of those in the dark-adapted samples on the same gel.

Serial tangential sectioning of the rat retinas. The method was used as described by Sokolov et al. (2002) and Strissel et al. (2006). Eyes were enucleated from light- or dark-adapted rats and dissected in ice-cold Ringer's solution under infrared light. The anterior portion of the eye was cut away, and the lens was removed. A $3 \mathrm{~mm}$ retina disc was cut from the center of the eyecup with a surgical trephine and transferred onto PVDF membrane with photoreceptors facing up. Retina was oriented on PVDF membrane inside of an open-face filter holder filled with the Ringer's solution. Extra liquid was removed by gentle suction applied from beneath the filter. The PVDF membrane with attached retina was then flat mounted between two glass slides separated by plastic spacers, clamped together with binder clips, frozen on dry ice, and sectioned on a cryomicrotome. The alignment of the retina surface with the cutting plane of the microtome knife was performed as described by Strissel et al. (2006). The 


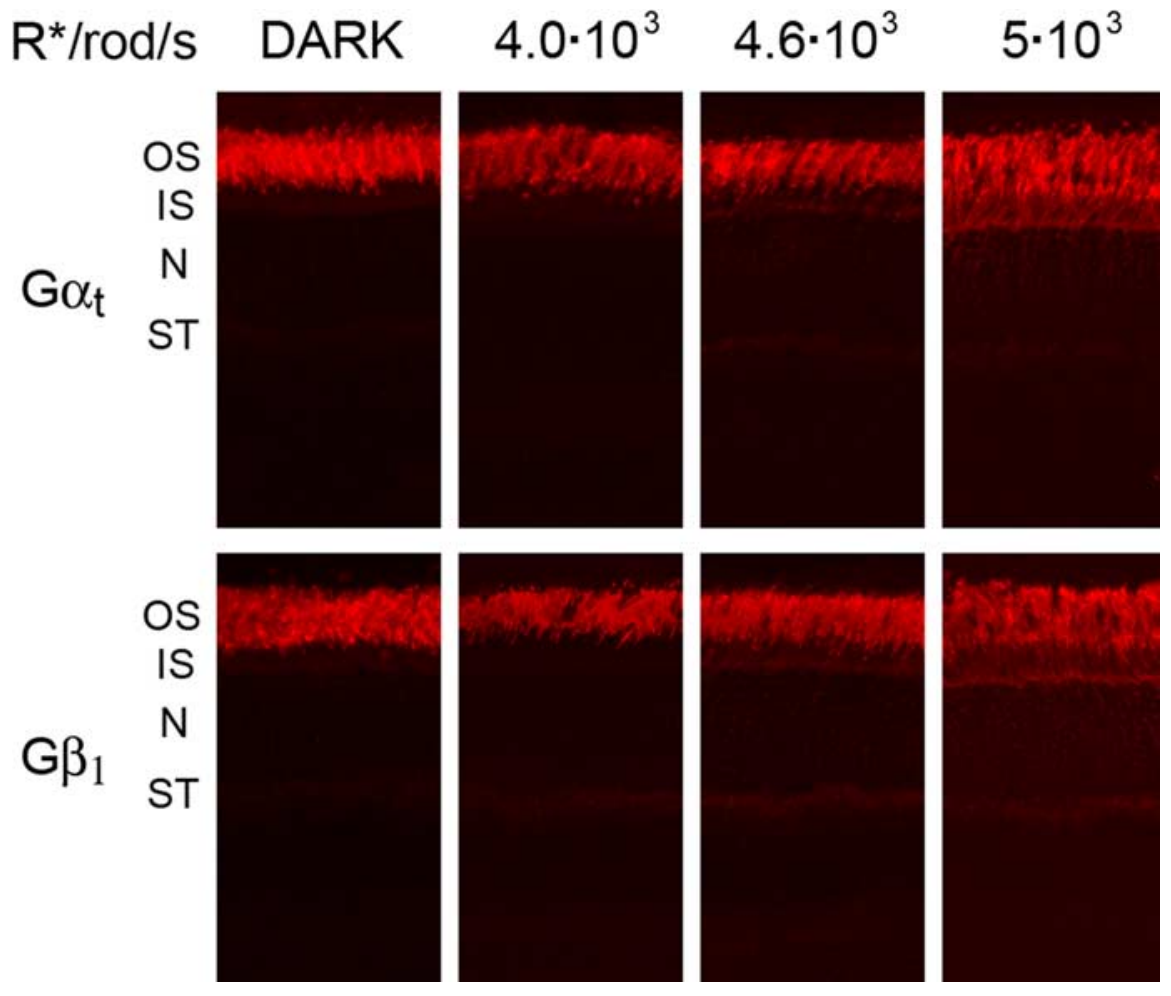

Figure 1. The light-dependent distribution of transducin subunits in wild-type mice. C57BL/6 mice were either dark adapted or exposed to 30 min of illumination from a calibrated light source producing photoexcited rhodopsin at initial rates indicated above each panel. The subcellular compartments of the rod are labeled as follows: $0 \mathrm{~S}$, outer segment; $I S$, inner segment; $\mathrm{N}$, nucleus; $\mathrm{ST}$, synaptic terminal. Note that significant changes in the inner segment and nuclear layer immunostaining become evident at the photoexcitation level of $4600 \mathrm{R}^{*} \cdot \mathrm{rod}^{-1} \cdot \mathrm{s}^{-1}$ and prominent at $5000 \mathrm{R}^{*} \cdot \mathrm{rod}^{-1} \cdot \mathrm{s}^{-1}$. The data are representative of the total of 11 mice analyzed at these and close light intensities.

edges of the retina were trimmed, $10 \mu \mathrm{m}$ serial tangential sections were collected, placed into precooled $1.5 \mathrm{ml}$ Eppendorf (Westbury, NY) tubes, and stored at $-80^{\circ} \mathrm{C}$ until used.

Transducin precipitation from the retina and retina sections. Affinitypurified polyclonal antibodies against $\mathrm{G} \alpha_{\mathrm{t}}$ were incubated with protein $\mathrm{G}$ agarose beads (GE Healthcare) in PBS buffer for $1 \mathrm{~h}$ at room temperature and then washed three times with PBS. We used 100-150 $\mu \mathrm{g}$ of antibodies per $20 \mu \mathrm{l}$ of beads for precipitation of transducin from retinas and $40-50 \mu \mathrm{g}$ of antibodies per $10 \mu \mathrm{l}$ of beads for precipitation of transducin from sections. Whole retinas or tangential sections were solubilized in $500 \mu \mathrm{l}$ of PBS containing 1.5\% Triton X-100 and Complete EDTA-free protease inhibitor cocktail (Roche Diagnostics, Indianapolis, IN), sonicated, vortexed, and spun down at 70,000 $\times g$ for $20 \mathrm{~min}$. Small aliquots of the supernatant were used for Western blot analysis of the protein distribution in sections, and the rest was added to the beads and incubated for $1 \mathrm{~h}$ at $4^{\circ} \mathrm{C}$ on an orbital shaker. After the incubation, $15 \mu \mathrm{l}$ of supernatant was collected for the analysis of unbound proteins, and beads were washed three times in PBS and boiled in $30 \mu$ lof the SDSPAGE sample buffer with $1 \%$ DTT for $10 \mathrm{~min}$. Twenty-five microliters of the extract were separated by SDS-PAGE for mass spectrometry (MS) analysis. Based on the comparison of Western blot staining of $\mathrm{G} \alpha_{\mathrm{t}}$ in the total and unbound fractions, the efficiency of transducin immunoprecipitation in all experiments was estimated to be $>90 \%$.

Mass spectrometry analysis of $G \alpha_{t} N$-terminal acylation. $\mathrm{G} \alpha_{\mathrm{t}}$ bands were excised from SDS-PAGE gels stained with colloidal Coomassie (Pierce Biotechnology) and trypsinized using the In-Gel Digestion kit (Pierce Biotechnology). The peptides were dissolved in $4 \mu \mathrm{l}$ of $50 \%$ acetonitrile, $0.1 \%$ trifluoroacetic acid, and $5 \mathrm{mg} / \mathrm{ml} \alpha$-4-hydroxycinnamic acid (Sigma) as a matrix for matrix-assisted laser desorption ionization (MALDI) mass spectrometry. The peptide-matrix mixture $(0.5 \mu \mathrm{l})$ was loaded onto a 192-spot MALDI plate and subjected to MALDI-time-offlight (TOF) MS and MS/MS analyses using the 4700 Proteomic Analyzer and the 4000 Series Explorer software (Applied Biosystems, Foster City, CA). Peaks with the molecular masses of 1001.50, 1025.51, 1027.52, and 1029.53 Da were reliably detected in the samples, which corresponded to a singly protonated N-terminal peptide of $\mathrm{G} \alpha_{\mathrm{t}}$, GAGASAEEK acylated with C12:0, C14:2, C14:1, or C14:0, respectively (Kokame et al., 1992; Neubert et al., 1992). The identity of these peptides was further confirmed by MS/MS analysis using collision-induced fragmentation.

The intensities of the peaks corresponding to the cluster area of each individual N-terminal peptide of $\mathrm{G} \alpha_{\mathrm{t}}$ were within the linear range of the MS peak intensities as calibrated with control peptides, angiotensin, bradykinin, and fibrinopeptide B and confirmed by serial dilutions of samples containing transducin peptides. To obtain an accurate calculation of the isotope cluster areas for the overlapping $1025.51,1027.52$, and 1029.53 peaks, the data generated by the 4000 Series Explorer software were corrected for a precise isotope peak ratio in the cluster calculated from an elemental formula of a modified peptide using an isotope ratio calculating tool within the Data Explorer software (Applied Biosystems).

\section{Results}

Light dependency of transducin translocation in wild-type and R9AP knock-out mice

A striking property of transducin translocation is that it is a steep nonlinear function of light intensity (Sokolov et al., 2002). Massive translocation in rods of living rats was observed only when the light intensity exceeded a critical level, and $\sim 90 \%$ of transducin was relocated from rod outer segments in response to a light of just twofold to threefold higher intensity. To understand the molecular mechanisms underlying this threshold, we studied the light dependency of transducin translocation in several models of genetically manipulated mice. Transducin distribution was detected using immunofluorescence staining of transducin subunits in retina cross sections. This technique is ubiquitously used in studies of protein translocation in photoreceptors. Although it does not allow quantitative determination of absolute amounts of transducin in individual subcellular compartments, it allows for reliable detection of major changes in transducin distribution, such as the massive translocation triggered at the critical light intensity level.

In the first series of experiments, we determined the minimum light intensity required for triggering massive transducin translocation in wild-type mice. Anesthetized animals were subjected to $30 \mathrm{~min}$ of illumination by light from a calibrated source, and the subcellular distribution of transducin subunits was analyzed by immunostaining of retina cross sections with antibodies recognizing $\mathrm{G} \alpha_{\mathrm{t}}$ and $\mathrm{G} \beta_{1}$ (Fig. 1). In the dark, nearly all immunostaining was confined to rod outer segments. The analysis of mice subjected to various illumination levels showed that this distribution remained unchanged until the light intensity at the cornea surface reached a critical level at $\sim 10$ scotopic $\mathrm{cd} / \mathrm{m}^{2}$, at which point prominent immunostaining was detected in rod inner segments and around rod nuclei. Based on measurements conducted with 11 mice, we found that this transition took place 
by illumination producing $\mathrm{R}^{\star}$ at the initial rate of $4300-5500 \mathrm{R}^{*} \cdot \operatorname{rod}^{-1} \cdot \mathrm{s}^{-1}$.

A study by Kerov et al. (2005) reported that the GTPase-deficient Q200L mutation in $\mathrm{G} \alpha_{\mathrm{t}}$ caused the majority of transducin subunits to localize outside rod outer segments regardless of conditions of illumination. They also showed that the translocation of $\mathrm{G} \alpha_{\mathrm{t}}$ in RGS9 knock-out mice, in which the lifetime of activated transducin is increased as a result of the decreased rate of its GTPase activity, occurs at a lower light intensity than in wildtype mice. We used a similar model of the R9AP knock-out mouse to determine whether the same shift occurs for $G \beta_{1} \gamma_{1}$ and to measure exactly how much the threshold was shifted. The R9AP knockout mouse lacks the entire GTPaseactivating complex (RGS9·G $\beta 5 \cdot R 9 A P$ ), and the physiological phenotype of these mice is identical to that of RGS9 knockouts (Keresztes et al., 2004). The analysis of 10 R9AP knock-out mice revealed a shift in the threshold required for massive translocation. Side-by-side analysis of wild-type and R9AP knock-out mice exposed to light producing $1200 \mathrm{R}^{*} \cdot \operatorname{rod}^{-1}$. $\mathrm{S}^{-1}$ revealed no reliable difference in immunostaining patterns (Fig. 2). However, illumination producing $2300 \mathrm{R}^{*} \cdot \operatorname{rod}^{-1}$. $\mathrm{s}^{-1}$ caused robust translocation of both transducin subunits in R9AP knock-outs but not in wild-type mice, as is particularly clear from the strong immunostaining around the nuclei. Overall, in R9AP knock-out mice, transducin translocation began at an $\sim 2.3$-fold lower light intensity $\left(1900-2300 \mathrm{R}^{*} \cdot \operatorname{rod}^{-1} \cdot \mathrm{s}^{-1}\right)$ than in wildtype animals.

\section{Light dependency of transducin translocation in W70A mice resembles that in R9AP knock-out mice}

The lack of the GTPase-activating complex increases both the lifetime of activated transducin and the magnitude of the downstream cellular response to light (Chen et al., 2000; Krispel et al., 2003; Keresztes et al., 2004). To distinguish which of these phenomena contributes to the shift in the threshold of transducin translocation in R9AP knock-out mice, we used the W70A mouse model, in which the lifetime of activated transducin is also prolonged, but the photoresponse is drastically diminished (Tsang et al., 1998). The W70A mutation in the transducin effector, cGMP phosphodiesterase $\gamma$ subunit (PDE $\gamma$ ), markedly reduces the affinity between G $\alpha_{\mathrm{t}}$ and PDE $\gamma$ (see also Otto-Bruc et al., 1993; Slepak et al., 1995). This impairs effector activation and eventually leads to an at least 200-fold reduction in the light sensitivity and a lack of photoresponse saturation at any light intensity (Tsang et al., 1998). The same mutation causes pro-
WT R9AP KO WT R9AP KO
$\mathrm{G} \alpha_{t}$


OS
Figure 2. Comparison of transducin subunit distribution in wild-type (WT) and R9AP knock-out (R9AP K0) mice. Animals were either dark adapted or exposed to $30 \mathrm{~min}$ of illumination producing photoexcited rhodopsin at initial rates indicated on the left of each panel. Mice subjected to light of each intensity were analyzed on the same day; their immunostaining was performed on the same slide and visualized using the same microscope settings. Note a major difference in immunostaining patterns at the light intensity producing $2300 \mathrm{R}^{*} \cdot \operatorname{rod}^{-1} \cdot \mathrm{S}^{-1}$. OS, Outer segment; IS, inner segment; N, nucleus; ST, synaptic terminal.

longed transducin activation, because RGS9 stimulates transducin GTPase most efficiently when transducin is bound to PDE $\gamma$ (Angleson and Wensel, 1994; Arshavsky et al., 1994; Tsang et al., 1998; Skiba et al., 2000).

We found that the light-dependency threshold of transducin translocation in W70A mice is shifted in the same direction as in R9AP knock-outs. The analysis of six W70A mice showed that transducin translocation is evident at a light intensity producing 


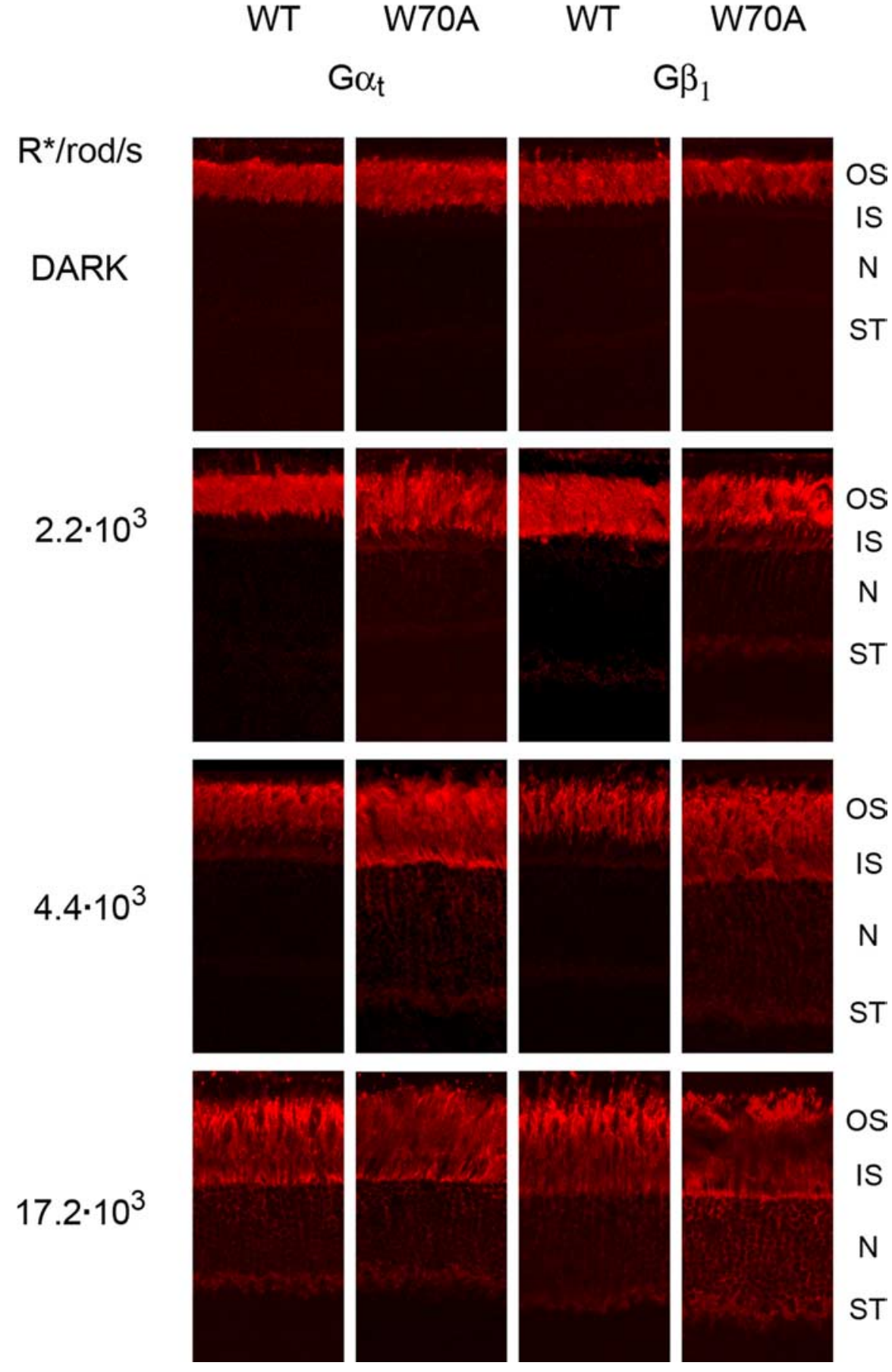

Figure 3. Comparison of transducin subunit distribution in wild-type (WT) and W70A mice. Animals were either dark adapted or exposed to 30 min of illumination producing photoexcited rhodopsin at initial rates indicated on the left of each panel. Note that the difference in immunostaining patterns becomes evident at the light intensity producing $2200 \mathrm{R}^{*} \cdot \mathrm{rod}^{-1} \cdot \mathrm{s}^{-1}$ and prominent at $4400 R^{*} \cdot \operatorname{rod}^{-1} \cdot S^{-1}$, when the translocation in the wild type just begins. OS, Outer segment; IS, inner segment; $N$, nucleus; ST, synaptic terminal.

$2200 \mathrm{R}^{*} \cdot \operatorname{rod}^{-1} \cdot \mathrm{s}^{-1}$. The difference between wild-type and W70A mice is even more striking at $4400 \mathrm{R}^{\star} \cdot \operatorname{rod}^{-1} \cdot \mathrm{s}^{-1}$, when the translocation in wild-type mice just begins (Fig. 3 ). Note that the W70A mice used in this study were albino, and for this reason, we used albino wild-type mice as side-by-side controls at all light intensities and directly measured the rate of rhodopsin bleaching in each animal type. This allowed us to describe the threshold in units of $\mathrm{R}^{*} \cdot \operatorname{rod}^{-1} \cdot \mathrm{s}^{-1}$, which most accurately reflects the intrinsic properties of rod photoreceptors.

\section{Phosducin dephosphorylation is insufficient for \\ transducin translocation}

The data from Figures 2 and 3 argue that the decrease in the rate of transducin GTPase correlates with the reduction of the light-dependency threshold of transducin translocation, regardless of whether the downstream signaling is enhanced (R9AP knock-out) or diminished (W70A mice). However, there is another mechanism that could potentially impact this threshold: light-dependent phosducin dephosphorylation. Phosducin is a $\mathrm{G} \beta \gamma$ interacting protein (Lee et al., 1987). Importantly, phosducin knock-out causes a reduced level of transducin translocation (Sokolov et al., 2004). Most likely, phosducin assists translocation by increasing the solubility of $\mathrm{G} \beta_{1} \gamma_{1}$ (Yoshida et al., 1994; Loew et al., 1998; Murray et al., 2001; Lee et al., 2004) and by preventing reassociation of transducin subunits into a heterotrimer after GTP hydrolysis. Phosducin is phosphorylated in the dark but dephosphorylated during illumination (Lee et al., 1990, 2004; Yoshida et al., 1994), and it is the nonphosphorylated form of phosducin that has the highest affinity for $\mathrm{G} \beta_{1} \gamma_{1}$ in vitro (Savage et al., 2000; Thulin et al., 2001). It has been suggested that phosducin dephosphorylation in light is an important regulatory event in transducin translocation (Lee et al., 2004).

To determine whether there is a correlation between phosducin phosphorylation status and the light-dependent threshold of transducin translocation, we assessed the status of phosducin phosphorylation using phospho-specific antibodies. The level of phosducin phosphorylation was measured at the light intensity of 4.5 scotopic $\mathrm{cd} / \mathrm{m}^{2}$, which triggers transducin translocation in R9AP knockout and W70A mice but not in wild-type animals (neither pigmented nor albino). Phosphorylation of the two principal light-regulated sites, serines 54 and 71, was detected using phospho-specific antibodies (see Materials and Methods) and compared with the level observed in completely dark-adapted mice.

These experiments did not reveal any clear correlation between the status of phosducin phosphorylation and the light intensity threshold for transducin translocation. Phosducin was nearly completely dephosphorylated in both wild-type and R9AP knock-out mice (Table 1), which indicates that phosducin dephosphorylation alone is not sufficient for triggering transducin translocation. However, massive transducin translocation took place in W70A mice, in which only a partial phosducin dephosphorylation was observed. The latter observation is consistent with the impaired activation of the phototransduction cascade in this mouse. This 
result does not necessarily challenge the very involvement of phosducin in transducin translocation, which is evident from reduced translocation of each transducin subunit in phosducin knock-out mice (Sokolov et al., 2004). Perhaps the partial dephosphorylation observed in W70A mice is sufficient for this function, or phosphorylation does not significantly affect phosducin affinity for $\mathrm{G} \beta_{1} \gamma_{1}$ in intact rods, in which both proteins are present at extremely high concentrations. These data provide a strong argument against phosducin dephosphorylation serving as the triggering event in transducin translocation and reinforce the hypothesis that the rate of transducin inactivation plays a crucial role in setting this threshold.

\section{Transducin translocation in mice} overexpressing the

GTPase-activating complex

If the position of the light-dependency threshold for transducin translocation is determined by the rate of transducin GTPase, then an increase of this rate should shift the threshold toward a brighter light level. We tested this prediction directly, by using the recently developed mouse model in which the expression level and the activity of the entire GTPase-activating complex is increased severalfold by the overexpression of R9AP (Krispel et al., 2006). No significant translocation of transducin was found in three mutant mice subjected to light intensities producing 5000-6000 $\mathrm{R}^{*}$. $\operatorname{rod}^{-1} \cdot s^{-1}$, which is sufficient to trigger translocation in wild-type animals (Fig. 4). However, translocation took place in three animals illuminated by brighter light producing $\sim 7000 \mathrm{R}^{*} \cdot \operatorname{rod}^{-1} \cdot \mathrm{s}^{-1}$. These data provide the most direct evidence that the threshold of transducin translocation is tightly regulated by the cellular capacity to inactivate transducin.

\section{The type of N-terminal acylation affects}

\section{$\mathrm{G} \boldsymbol{\alpha}_{\mathrm{t}}$ translocation}

In the last set of experiments, we addressed another factor that may affect the translocation of transducin in light, its interaction with membranes of photoreceptor discs. These interactions are thought to be determined primarily by the nature of their lipid modifications. We focused on $\mathrm{G} \alpha$, which in rods is modified with one of four N-terminal lipid moieties: lauroyl (C12:0), myristoyl (C14:0), (cis- $\left.\Delta^{5}\right)$-tetradecaenoyl (C14:1), or (cis-,cis- $\left.\Delta^{5}, \Delta^{8}\right)$ tetradecadienoyl (C14:2) (Kokame et al., 1992; Neubert et al., 1992; Neubert and Hurley, 1998). These modifications have different lipophilic properties, with C14:0 being the most lipophilic, followed by C14:1, C12:0, and C14:2 (Peitzsch and McLaughlin, 1993). This natural heterogeneity enabled us to establish the relationship between the lipophilic properties of individual $\mathrm{G} \alpha$ molecules and the efficiency of their movement from rod outer segments in light.

First, we confirmed the presence of multiple acyl residues on $\mathrm{G} \alpha_{\mathrm{t}}$ and determined their relative abundance in rodent rods, because all published studies of heterogeneous $\mathrm{G} \alpha_{\mathrm{t}}$ acylation were performed with bovine transducin. We used rats rather than mice, because the larger size of their retinas produced higher
Table 1. Light-evoked dephosphorylation of phosducin in the retinas of W70A and R9AP knock-out mice

\begin{tabular}{llll}
\hline & Control $^{a}$ & W70A & R9AP K0 \\
\hline Serine 54 & 0 & $76 \pm 6$ & $5 \pm 1$ \\
Serine 71 & 0 & $51 \pm 5$ & $9 \pm 3$
\end{tabular}

Anesthetized mice were exposed to light ( $4.5 \mathrm{scotopic} \mathrm{cd} / \mathrm{m}^{2}$ at the cornea surface) for $30 \mathrm{~min}$, which triggered transducin translocation in R9AP knock-out (KO) and W70A mice but not wild-type mice. Eyeballs were rapidly extracted and frozen, and the status of phosducin phosphorylation was determined as described in Materials and Methods. Data are expressed as a percentage of the level in the dark-adapted retina (mean $\pm \mathrm{SEM} ; n=6$ ).

${ }^{a}$ Both $\mathrm{CD}-1$ and $\mathrm{C} 57 \mathrm{BL} / 6$ mice were analyzed.
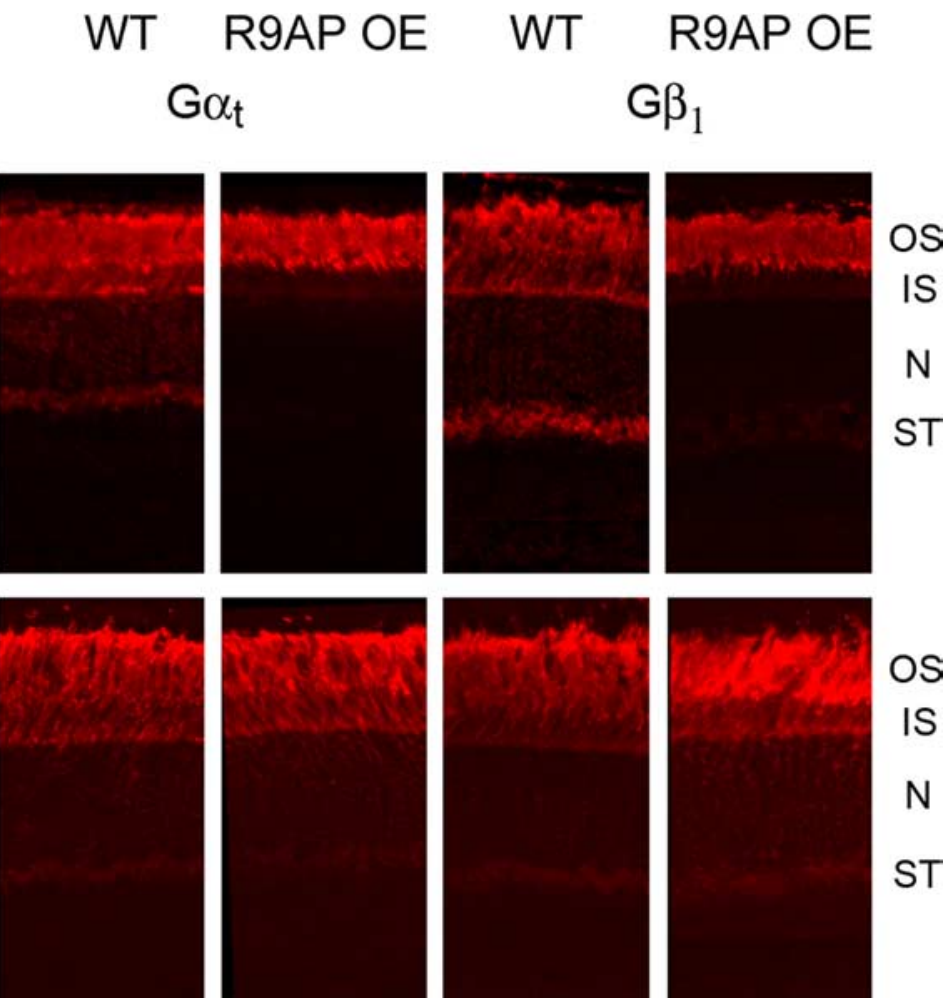

\begin{tabular}{llll}
\hline $\mathrm{C14}: 0$ & $\mathrm{C14:1}$ & $\mathrm{C12:0}$ & $\mathrm{C14:2}$ \\
\hline $8.2 \pm 2.1$ & $15.9 \pm 1.5$ & $20.2 \pm 1.7$ & $55.7 \pm 5.3$
\end{tabular}

The amount of each acylated isoform was determined on the basis of the isotope cluster areas of individually acylated N-terminal peptides quantified by MALDI-TOF mass spectrometry. The fraction of each isoform is expressed as a percentage of the total (mean $\pm S D ; n=2$ ).

yields of transducin. $\mathrm{G} \alpha_{\mathrm{t}}$ was immunoprecipitated from homogenized retinas, separated by SDS-PAGE, and digested by trypsin, and the resulting peptides (including acylated $\mathrm{N}$-terminal peptides) were analyzed by MALDI-TOF mass spectrometry as described in Materials and Methods. All four acyl modifications were detected (Table 2), and their relative amounts were similar to the distributions reported for bovine transducin (Kokame et al., 1992; Neubert et al., 1992; Neubert and Hurley, 1998), with the exception that the fraction of $\mathrm{C} 14: 2$ in the rat was approximately twice as large. We believe that this difference does not reflect a variation in the mass spectrometry protocols, because our own mass spectrometry analysis of purified bovine transdu- 
Dark

A

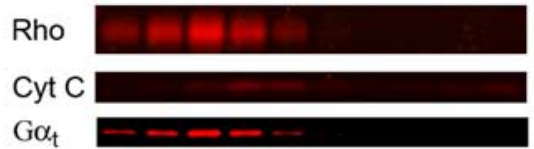

B

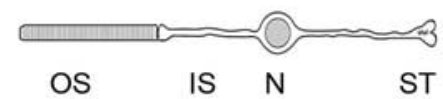

C $\quad \mathrm{IgG} / \mathrm{HC}$

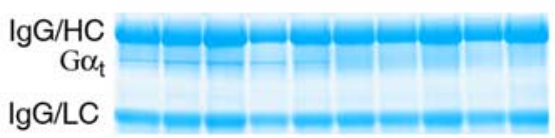

D

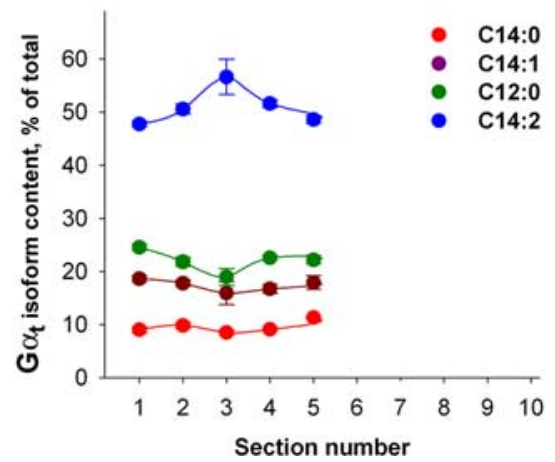

Light
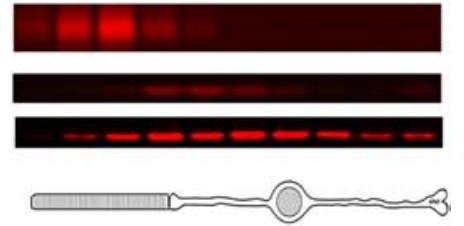

OS

IS

$\mathrm{N}$ ST
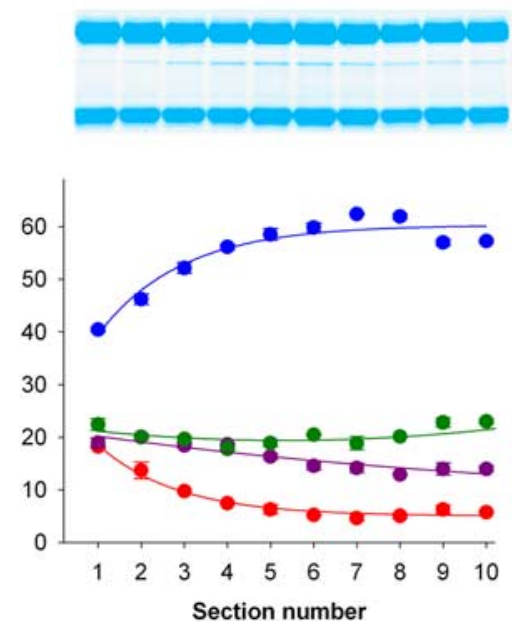

Figure 5. The dependency of $\mathrm{G} \alpha_{\mathrm{t}}$ translocation on the type of its $\mathrm{N}$-terminal acylation in dark-adapted (left) and light-adapted (right) rats. Anesthetized animals were kept in the dark or exposed to $40 \mathrm{~min}$ of illumination $\left(240 \mathrm{scotopic} \mathrm{cd} / \mathrm{m}^{2}\right.$ at the cornea surface), and their retinas were extracted and prepared for serial tangential sectioning (see Materials and Methods). Sections representing the same subcellular compartments were pooled from four dark-adapted and five light-adapted retinas. $\boldsymbol{A}$, The distribution of $\mathrm{G} \alpha_{\mathrm{t}}$ and two intracellular markers, rhodopsin (Rho) and cytochrome C (Cyt C), was analyzed in small aliquots by Western blotting. $\boldsymbol{B}$, A schematic drawing of a rod cell. OS, Outer segment; IS, inner segment; N, nucleus; ST, synaptic terminal. $\boldsymbol{C}$, $\mathrm{G} \alpha_{\mathrm{t}}$ was immunoprecipitated from each pool of the sections and separated from coeluting $\lg \mathrm{G}$ heavy $(\mathrm{lg} \mathrm{G} / \mathrm{HC})$ and light $(\mathrm{IgG} / \mathrm{LC})$ chains by SDS-PAGE. $D$, The fraction of each acylated isoform of $\mathrm{G} \alpha$ is expressed as a percentage of the total of all isoforms. The amount of each isoform was determined on the basis of the isotope cluster areas of individually acylated $\mathrm{N}$-terminal peptides quantified by MALDI-TOF mass spectrometry. The data are taken from two independent mass spectrometry determinations, with error bars representing the scatter of the data.

cin yielded the corresponding distribution, very close to that from the most recent report by Neubert and Hurley (1998) (22/ 31/18/30 C14:0/14:1/12:0/14:2 in our experiments vs $21 / 34 / 12 / 33$ in theirs).

To study the relationship between the type of lipid modification and the extent of $\mathrm{G} \alpha_{\mathrm{t}}$ translocation in light, we developed an approach combining microdissection of the photoreceptor layer of the retina with mass spectrometric analysis of transducin acylation in individual subcellular compartments of the rod. Progressive $10 \mu \mathrm{m}$ tangential sections were obtained from the photoreceptor layer of flat-mounted frozen retinas of several rats. Sections from multiple retinas representing the same part of the rod cell were pooled and solubilized in detergent. $\mathrm{G} \alpha_{\mathrm{t}}$ was immunoprecipitated from each pool, and the amount of each acylated isoform was determined by mass spectrometry.

We first established the distribution of variably acylated $\mathrm{G} \alpha_{\mathrm{t}}$ isoforms in dark-adapted rats. In the dark, $\mathrm{G} \alpha_{\mathrm{t}}$ was detected only in the five distal sections, which also contained rhodopsin, verifying that those sections represent rod outer segments (Fig. $5 A-C$, left). The four individually acylated isoforms of $\mathrm{G} \alpha_{\mathrm{t}}$ were evenly represented throughout these sections, with no enrichment observed at either the proximal or distal end of the outer segment (Fig. 5D, left). We next analyzed $\mathrm{G} \alpha_{\mathrm{t}}$ isoform distribution in rats exposed to bright light that caused maximal transducin translocation. In this case, $\mathrm{G} \alpha_{\mathrm{t}}$ was reliably detected through- out the entire length of the rod cell, with the majority present in the inner segment (Fig. 5A-C, right). Although each acylated isoform was found in every part of the cell, their relative abundance varied (Fig. 5D, right). The fraction of $\mathrm{G} \alpha_{\mathrm{t}}$ modified by the most lipophilic C14:0 was gradually reduced by approximately fourfold from the most distal outer segment section toward the sections representing other subcellular compartments. A small reduction in the same direction was observed for C14:1 C12:0 was evenly represented throughout the entire cell. The fraction of $\mathrm{G} \alpha_{\mathrm{t}}$ modified by the least lipophilic C14:2 was $\sim 1.5$ fold enriched in the non-outer segment sections.

These experiments demonstrate that neither modification assures complete translocation of $\mathrm{G} \alpha_{\mathrm{t}}$ or its complete retention in the outer segment. However, the less lipophilic the acyl modification is, the higher the efficiency of $\mathrm{G} \alpha_{\mathrm{t}}$ translocation from the outer segment is. These data indicate that $\mathrm{G} \alpha_{\mathrm{t}}$ interactions with membranes of the photoreceptor discs affect the rate at which transducin escapes the outer segment during illumination.

\section{Discussion}

Light-driven transducin translocation was discovered two decades ago (Brann and Cohen, 1987; Philp et al., 1987; Whelan and McGinnis, 1988) but was viewed by many investigators as an artifact (Roof and Heth, 1988) until it was analyzed quantitatively and shown to contribute to rod light adaptation (Sokolov et al., 2002). Lately, most studies have focused on elucidating whether transducin translocates by diffusion or is carried by molecular motors (for review, see Calvert et al., 2006). Much less attention has been given to another striking mechanistic feature of transducin translocation, its light-dependency threshold. Here, we demonstrated that this threshold is determined by the capacity of the GTPase-activating complex to inactivate transducin and that the efficiency of $\mathrm{G} \alpha_{\mathrm{t}}$ translocation is dependent on the hydrophobicity of its acyl modification. In this section, we connect these findings in a mechanistic model and discuss the potential significance of the translocation threshold in rod adaptation to bright light.

\section{Transducin translocation takes place in three major steps}

In the dark, the transducin heterotrimer is localized predominantly in rod outer segments, in which it is tightly associated with photoreceptor disc membranes as a result of the combined action of two lipid modifications: an acyl group on $\mathrm{G} \alpha_{\mathrm{t}}$ (Kokame et al., 1992; Neubert et al., 1992) and a farnesyl group on $\mathrm{G} \gamma_{1}$ (Fukada et al., 1990; Lai et al., 1990). The growing consensus is that transducin translocation is accomplished in three principal steps (Kerov et al., 2005). The first step is transducin activation by photoexcited rhodopsin (Mendez et al., 2003), which causes the separation of $\mathrm{G} \alpha_{\mathrm{t}}$ and $\mathrm{G} \beta_{1} \gamma_{1}$ subunits (Fung et al., 1981). Next, each subunit dissociates from the membrane to the cytosol, be- 
cause each contains only one lipid modification providing a much lower membrane affinity than the two modifications in the heterotrimer (Seitz et al., 1999). Finally, transducin subunits escape from the outer segment separately from one another, as evident from the difference in their translocation kinetics (Sokolov et al., 2002; Kassai et al., 2005; Calvert et al., 2006). Most likely, they diffuse through the rod cytoplasm (Sokolov et al., 2004; Nair et al., 2005), although a few studies suggest the involvement of molecular motors (McGinnis et al., 2002; Peterson et al., 2005).

\section{The threshold for transducin translocation is determined by the balance between the rate of transducin dissociation from membranes and the capacity of the GTPase-activating complex} Remarkably, none of the three translocation steps suggests the existence of a light-dependency threshold. Our experiments with W70A mice and the analysis of phosducin phosphorylation indicate that the threshold is not set by signaling events downstream from transducin. However, our experiments do provide a strong correlation between the threshold light intensity for translocation and the capacity of the GTPase-activating complex to inactivate transducin. The threshold was reduced in the R9AP knockout and W70A mice, in which the rate of transducin inactivation was diminished, and increased in R9AP overexpressors, in which the rate of transducin inactivation was enhanced.

This is consistent with the following model illustrated in Figure 6. Under relatively dim illumination, the rate at which transducin is inactivated by RGS9 is much higher than the rate at which transducin subunits dissociate from the membranes. However, at a certain light level, the amount of activated transducin exceeds the capacity of RGS9 to readily inactivate it. The lifetime of this "additional" transducin rises sharply (because normally RGS9 activates transducin GTPase by $\sim 100$-fold), which produces a significant fraction of $\mathrm{G} \alpha_{\mathrm{t}}$ and $\mathrm{G} \beta_{1} \gamma_{1}$ that stay apart sufficiently long to dissociate from the membranes before transducin heterotrimer is re-formed.

Is there a reason to believe that rods do not have a sufficient amount of RGS9 to serve the entire content of transducin? One argument is that rods have $\sim 60$-fold less RGS9 than transducin (Zhang et al., 2003). However, the strongest evidence comes from electrophysiological studies. In rods, the photocurrent recovery time after a series of saturating flashes remains a linear function of the natural logarithm of flash intensity over an extended illumination range (Pepperberg et al., 1992). The slope of this plot, known as the "Pepperberg plot," was recently shown to represent the rate at which $\mathrm{G} \alpha_{\mathrm{t}} \cdot \mathrm{GTP}$ is inactivated by RGS9 (Krispel et al., 2006). Importantly, this linear relationship "breaks" at a critical light level, after which the rod stays excited much longer, which means that the amount of transducin produced by this or brighter light exceeds the capacity of RGS9 to efficiently control its GTPase activity. (Note that the light intensity for the threshold of transducin translocation cannot be directly compared with the breaking point of the Pepperberg plot because of intrinsic differences in experimental paradigms: prolonged continuous illumination vs short bright flashes.)

This logic may also be relevant to understanding why transducin translocation was not detected in cones (Elias et al., 2004; Kennedy et al., 2004; Coleman and Semple-Rowland, 2005). Cones contain more RGS9 than rods (Cowan et al., 1998), which is expected to result in a faster transducin inactivation and therefore its better retention on the membranes. However, other reasons, such as a shorter lifetime of photoexcited cone pigment or
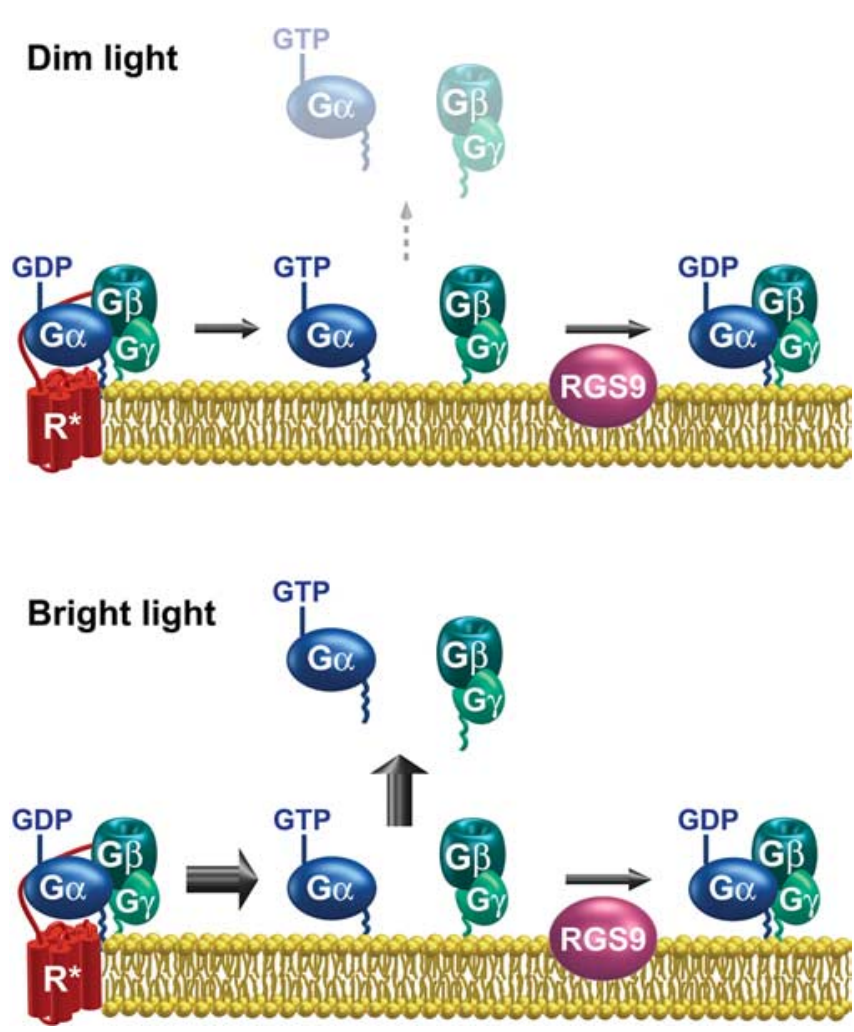

Figure 6. The capacity of the RGS9 GTPase-activating complex determines the light intensity threshold of transducin translocation. The amount of activated transducin in each case is encoded by the thickness of arrows. Top, At the light intensity below threshold, activated transducin is quickly inactivated by RGS9, providing little time for dissociating from the membrane. Bottom, At the light intensity above threshold, more transducin is activated than could be readily inactivated by RGS9, and a fraction of transducin stays activated sufficiently long to dissociate from the membrane to the cytosol. The diagram is simplified by not illustrating the reversibility of transducin membrane association, $\mathrm{G} \alpha_{\mathrm{t}}$ interaction with cGMP phosphodiesterase, and $\mathrm{G} \beta_{1} \gamma_{1}$ interaction with phosducin, each likely to affect specific parameters of the membrane/cytosol distribution of transducin. The basic elements of this diagram are reproduced with permission from Calvert et al. (2006).

different pattern of lipid modification, should be considered as well.

\section{The hydrophobicity of $\mathrm{G} \boldsymbol{\alpha}_{\mathrm{t}}$ acyl modification affects the translocation efficiency}

Our model assumes that the dissociation of transducin subunits from membranes to cytosol imposes a limitation on transducin translocation. This is generally consistent with the rate of transducin translocation in saturating light (Sokolov et al., 2002) being three to six times slower than the rate at which a soluble GFP (green fluorescent protein) derivative diffuses between the inner and the outer segments of mouse rods (Nair et al., 2005). The ability of transducin subunits to dissociate from the disc membranes is expected to be determined primarily by the nature of their lipid modifications. Indeed, Kassai et al. (2005) found that substitution of the farnesyl residue in $\mathrm{G} \gamma_{1}$ with a more lipophilic geranylgeranyl moiety impairs $\mathrm{G} \beta_{1} \gamma_{1}$ translocation despite a normal translocation of $\mathrm{G} \alpha$.

We focused on $\mathrm{G} \alpha$, which can be modified by one of four different acyl moieties in the same cell, thus providing a unique opportunity to establish the relationship between the translocation efficiency and the hydrophobicity of these modifications. We found that none of the modifications, even the most lipophilic, C14:0, provided sufficient membrane affinity to pre- 
vent $\mathrm{G} \alpha_{\mathrm{t}}$ from translocating. However, the extent of translocation varied among the various acylated $\mathrm{G} \alpha$ t molecules, with more lipophilic isoforms translocating to a lesser extent than the less lipophilic isoforms. This fine dependency of the translocation efficiency on the lipophilic properties of $\mathrm{G} \alpha_{\mathrm{t}}$ supports the idea that $\mathrm{G} \alpha_{\mathrm{t}}$ dissociation from the disc membranes (and perhaps secondary interactions with disc membranes after translocation) limits the rate at which $\mathrm{G} \alpha_{\mathrm{t}}$ escapes rod outer segments during illumination.

\section{The threshold in transducin translocation: for adaptation or neuroprotection?}

Our measurements indicate that transducin translocation is triggered by light producing $4300-5500 \mathrm{R}^{*} \cdot \operatorname{rod}^{-1} \cdot \mathrm{s}^{-1}$ [which is a somewhat lower photoexcitation rate than that calculated indirectly from the luminance on the cornea surface by Sokolov et al. (2002)]. Remarkably, this fits perfectly into the 4000-6000 $\mathrm{R}^{\star}$. $\operatorname{rod}^{-1} \cdot \mathrm{s}^{-1}$ range known to completely saturate mammalian rods under experimental conditions not allowing sufficient time for transducin translocation (Nakatani et al., 1991). This makes it plausible that both phenomena, transducin translocation and response saturation, occur for essentially the same reason, the inability of rods to control the rate of transducin GTPase beyond a certain light intensity. In this context, transducin translocation can be viewed as an elegant self-regulating mechanism triggered at the point at which the rod exhausts other means of staying away from response saturation.

The functional consequences of this self-regulation could be severalfold. Because the reduction of transducin concentration in rod outer segments causes the reduction of signal amplification in the phototransduction cascade (Sokolov et al., 2002), massive translocation of transducin may extend the range of the light responsiveness of the rod beyond the limits imposed by other adaptation mechanisms. This may explain why psychophysical experiments estimate that rods continue to signal at $>2000$ scotopic trolands (for review, see Makous, 2001), a level that produces $>17,000 \mathrm{R}^{\star} \cdot \operatorname{rod}^{-1} \cdot \mathrm{s}^{-1}$ (Kraft et al., 1993) and completely saturates rods in conventional electrophysiological experiments.

On the other hand, rod saturation marks the transition from mesopic to cone-dominated photopic vision. Under these conditions, rods contribute little to vision, and transducin translocation may prevent excessive energy consumption by rods by reducing the number of transducin molecules undergoing the cycle of activation/inactivation [for estimates of energy saving by this mechanism, see Burns and Arshavsky (2005)]. This may reduce the metabolic stress in the retina, commonly believed to contribute to pathological processes in the eye. In addition, a reduced level of cellular signaling caused by transducin translocation may reduce the chance of apoptotic death of the rod. At least in rodents, some forms of apoptosis are suggested to be caused by excessive signaling through the phototransduction cascade (Fain, 2006). Finally, when vision is dominated by cones, rods may perform more of their housekeeping functions, such as checking the integrity of transducin subunits by the ubiquitin proteasome system located in the inner segments (Obin et al., 2002).

\section{References}

Angleson JK, Wensel TG (1994) Enhancement of rod outer segment GTPase accelerating protein activity by the inhibitory subunit of cGMP phosphodiesterase. J Biol Chem 269:16290-16296.

Arshavsky VY, Dumke CL, Zhu Y, Artemyev NO, Skiba NP, Hamm HE, Bownds MD (1994) Regulation of transducin GTPase activity in bovine rod outer segments. J Biol Chem 269:19882-19887.
Brann MR, Cohen LV (1987) Diurnal expression of transducin mRNA and translocation of transducin in rods of rat retina. Science 235:585-587.

Burns ME, Arshavsky VY (2005) Beyond counting photons: trials and trends in vertebrate visual transduction. Neuron 48:387-401.

Calvert PD, Strissel KJ, Schiesser WE, Pugh Jr EN, Arshavsky VY (2006) Light-driven translocation of signaling proteins in vertebrate photoreceptors. Trends Cell Biol 16:560-568.

Chen CK, Burns ME, He W, Wensel TG, Baylor DA, Simon MI (2000) Slowed recovery of rod photoresponse in mice lacking the GTPase accelerating protein RGS9-1. Nature 403:557-560.

Coleman JE, Semple-Rowland SL (2005) GC1 deletion prevents lightdependent arrestin translocation in mouse cone photoreceptor cells. Invest Ophthalmol Vis Sci 46:12-16.

Cowan CW, Fariss RN, Sokal I, Palczewski K, Wensel TG (1998) High expression levels in cones of RGS9, the predominant GTPase accelerating protein of rods. Proc Natl Acad Sci USA 95:5351-5356.

Elias RV, Sezate SS, Cao W, McGinnis JF (2004) Temporal kinetics of the light/dark translocation and compartmentation of arrestin and alphatransducin in mouse photoreceptor cells. Mol Vis 10:672-681.

Fain GL (2006) Why photoreceptors die (and why they don't). BioEssays 28:344-354.

Fukada Y, Takao T, Ohguro H, Yoshizawa T, Akino T, Shimonishi Y (1990) Farnesylated gamma-subunit of photoreceptor $\mathrm{G}$ protein indispensable for GTP-binding. Nature 346:658-660.

Fung BK, Hurley JB, Stryer L (1981) Flow of information in the lighttriggered cyclic nucleotide cascade of vision. Proc Natl Acad Sci USA 78:152-156.

Giessl A, Pulvermuller A, Trojan P, Park JH, Choe HW, Ernst OP, Hofmann KP, Wolfrum U (2004) Differential expression and interaction with the visual G-protein transducin of centrin isoforms in mammalian photoreceptor cells. J Biol Chem 279:51472-51481.

Heck M, Hofmann KP (2001) Maximal rate and nucleotide dependence of rhodopsin-catalyzed transducin activation: initial rate analysis based on a double displacement mechanism. J Biol Chem 276:10000-10009.

Kassai H, Aiba A, Nakao K, Nakamura K, Katsuki M, Xiong WH, Yau KW, Imai H, Shichida Y, Satomi Y, Takao T, Okano T, Fukada Y (2005) Farnesylation of retinal transducin underlies its translocation during light adaptation. Neuron 47:529-539.

Kennedy MJ, Dunn FA, Hurley JB (2004) Visual pigment phosphorylation but not transducin translocation can contribute to light adaptation in zebrafish cones. Neuron 41:915-928.

Keresztes G, Martemyanov KA, Krispel CM, Mutai H, Yoo PJ, Maison SF, Burns ME, Arshavsky VY, Heller S (2004) Absence of the RGS9•G $\beta 5$ GTPase-activating complex in photoreceptors of the R9AP knockout mouse. J Biol Chem 279:1581-1584.

Kerov V, Chen D, Moussaif M, Chen YJ, Chen CK, Artemyev NO (2005) Transducin activation state controls its light-dependent translocation in rod photoreceptors. J Biol Chem 280:41069-41076.

Kokame K, Fukada Y, Yoshizawa T, Takao T, Shimonishi Y (1992) Lipid modification at the $\mathrm{N}$ terminus of photoreceptor $\mathrm{G}$-protein $\alpha$-subunit. Nature 359:749-752.

Kraft TW, Schneeweis DM, Schnapf JL (1993) Visual transduction in human rod photoreceptors. J Physiol (Lond) 464:747-765.

Krispel CM, Chen CK, Simon MI, Burns ME (2003) Prolonged photoresponses and defective adaptation in rods of $\mathrm{G} \beta 5-/-$ mice. J Neurosci 23:6965-6971.

Krispel CM, Chen D, Melling N, Chen YJ, Martemyanov KA, Quillinan N, Arshavsky VY, Wensel TG, Chen CK, Burns ME (2006) RGS expression rate-limits recovery of rod photoresponses. Neuron 51:409-416.

Lai RK, Perez-Sala D, Canada FJ, Rando RR (1990) The gamma subunit of transducin is farnesylated. Proc Natl Acad Sci USA 87:7673-7677.

Lee BY, Thulin CD, Willardson BM (2004) Site-specific phosphorylation of phosducin in intact retina. Dynamics of phosphorylation and effects on $G$ protein $\beta \gamma$ dimer binding. J Biol Chem 279:54008-54017.

Lee RH, Lieberman BS, Lolley RN (1987) A novel complex from bovine visual cells of a 33,000-dalton phosphoprotein with beta- and gammatransducin: purification and subunit structure. Biochemistry 26:3983-3990.

Lee RH, Brown BM, Lolley RN (1990) Protein kinase A phosphorylates retinal phosducin on serine 73 in situ. J Biol Chem 265:15860-15866.

Loew A, Ho YK, Blundell T, Bax B (1998) Phosducin induces a structural change in transducin beta gamma. Structure 6:1007-1019. 
Lyubarsky AL, Daniele LL, Pugh Jr EN (2004) From candelas to photoisomerizations in the mouse eye by rhodopsin bleaching in situ and the light-rearing dependence of the major components of the mouse ERG. Vision Res 44:3235-3251.

Makous W (2001) Scotopic vision. In: Visual neurosciences (Chalupa LM, Werner JH, eds), pp 215-233. Cambridge, MA: MIT.

McGinnis JF, Matsumoto B, Whelan JP, Cao W (2002) Cytoskeleton participation in subcellular trafficking of signal transduction proteins in rod photoreceptor cells. J Neurosci Res 67:290-297.

Mendez A, Lem J, Simon M, Chen J (2003) Light-dependent translocation of arrestin in the absence of rhodopsin phosphorylation and transducin signaling. J Neurosci 23:3124-3129.

Murray D, McLaughlin S, Honig B (2001) The role of electrostatic interactions in the regulation of the membrane association of $\mathrm{G}$ protein $\beta \gamma$ heterodimers. J Biol Chem 276:45153-45159.

Nair KS, Hanson SM, Mendez A, Gurevich EV, Kennedy MJ, Shestopalov VI, Vishnivetskiy SA, Chen J, Hurley JB, Gurevich VV, Slepak VZ (2005) Light-dependent redistribution of arrestin in vertebrate rods is an energyindependent process governed by protein-protein interactions. Neuron 46:555-567.

Nakatani K, Tamura T, Yau KW (1991) Light adaptation in retinal rods of the rabbit and two other nonprimate mammals. J Gen Physiol 97:413-435.

Neubert TA, Hurley JB (1998) Functional heterogeneity of transducin alpha subunits. FEBS Lett 422:343-345.

Neubert TA, Johnson RS, Hurley JB, Walsh KA (1992) The rod transducin alpha subunit amino terminus is heterogeneously fatty acylated. J Biol Chem 267:18274-18277.

Obin M, Lee BY, Meinke G, Bohm A, Lee RH, Gaudet R, Hopp JA, Arshavsky VY, Willardson BM, Taylor A (2002) Ubiquitylation of the transducin $\beta \gamma$ subunit complex. Regulation by phosducin. J Biol Chem 277:44566-44575.

Otto-Bruc A, Antonny B, Vuong TM, Chardin P, Chabre M (1993) Interaction between the retinal cyclic GMP phosphodiesterase inhibitor and transducin. Kinetics and affinity studies. Biochemistry 32:8636-8645.

Peitzsch RM, McLaughlin S (1993) Binding of acylated peptides and fatty acids to phospholipid vesicles: pertinence to myristoylated proteins. Biochemistry 32:10436-10443.

Pepperberg DR, Cornwall MC, Kahlert M, Hofmann KP, Jin J, Jones GJ, Ripps H (1992) Light-dependent delay in the falling phase of the retinal rod photoresponse. Vis Neurosci 8:9-18.

Peterson JJ, Orisme W, Fellows J, McDowell JH, Shelamer CL, Dugger DR, Smith WC (2005) A role for cytoskeletal elements in the light-driven translocation of proteins in rod photoreceptors. Invest Ophthalmol Vis Sci 46:3988-3998.

Philp NJ, Chang W, Long K (1987) Light-stimulated protein movement in rod photoreceptor cells of the rat retina. FEBS Lett 225:127-132.

Pulvermuller A, Giessl A, Heck M, Wottrich R, Schmitt A, Ernst OP, Choe
HW, Hofmann KP, Wolfrum U (2002) Calcium-dependent assembly of centrin-G-protein complex in photoreceptor cells. Mol Cell Biol 22:2194-2203.

Roof DJ, Heth CA (1988) Expression of transducin in retinal rod photoreceptor outer segments. Science 241:845-847.

Savage JR, McLaughlin JN, Skiba NP, Hamm HE, Willardson BM (2000) Functional roles of the two domains of phosducin and phosducin-like protein. J Biol Chem 275:30399-30407.

Seitz HR, Heck M, Hofmann KP, Alt T, Pellaud J, Seelig A (1999) Molecular determinants of the reversible membrane anchorage of the G-protein transducin. Biochemistry 38:7950-7960.

Skiba NP, Hopp JA, Arshavsky VY (2000) The effector enzyme regulates the duration of $\mathrm{G}$ protein signaling in vertebrate photoreceptors by increasing the affinity between transducin and RGS protein. J Biol Chem 275:32716-32720.

Slepak VZ, Artemyev NO, Zhu Y, Dumke CL, Sabacan L, Sondek J, Hamm HE, Bownds MD, Arshavsky VY (1995) An effector site that stimulates G-protein GTPase in photoreceptors. J Biol Chem 270:14319-14324.

Sokolov M, Lyubarsky AL, Strissel KJ, Savchenko AB, Govardovskii VI, Pugh Jr EN, Arshavsky VY (2002) Massive light-driven translocation of transducin between the two major compartments of rod cells: a novel mechanism of light adaptation. Neuron 34:95-106.

Sokolov M, Strissel KJ, Leskov IB, Michaud NA, Govardovskii VI, Arshavsky VY (2004) Phosducin facilitates light-driven transducin translocation in rod photoreceptors. Evidence from the phosducin knockout mouse. J Biol Chem 279:19149-19156.

Strissel KJ, Sokolov M, Trieu LH, Arshavsky VY (2006) Arrestin translocation is induced at a critical threshold of visual signaling and is superstoichiometric to bleached rhodopsin. J Neurosci 26:1146-1153.

Thulin CD, Savage JR, McLaughlin JN, Truscott SM, Old WM, Ahn NG, Resing KA, Hamm HE, Bitensky MW, Willardson BM (2001) Modulation of the $\mathrm{G}$ protein regulator phosducin by $\mathrm{Ca}^{2+} /$ calmodulindependent protein kinase II phosphorylation and 14-3-3 protein binding. J Biol Chem 276:23805-23815.

Ting TD, Goldin SB, Ho Y-K (1993) Purification and characterization of bovine transducin and its subunits. Methods Neurosci 15:180-195.

Tsang SH, Burns ME, Calvert PD, Gouras P, Baylor DA, Goff SP, Arshavsky VY (1998) Role for the target enzyme in deactivation of photoreceptor G protein in vivo. Science 282:117-121.

Whelan JP, McGinnis JF (1988) Light-dependent subcellular movement of photoreceptor proteins. J Neurosci Res 20:263-270.

Yoshida T, Willardson BM, Wilkins JF, Jensen GJ, Thornton BD, Bitensky MW (1994) The phosphorylation state of phosducin determines its ability to block transducin subunit interactions and inhibit transducin binding to activated rhodopsin. J Biol Chem 269:24050-24057.

Zhang X, Wensel TG, Kraft TW (2003) GTPase regulators and photoresponses in cones of the eastern chipmunk. J Neurosci 23:1287-1297. 